\title{
Idiopathische Skoliose - Ätiologie, Klassifikation und Therapie
}

\author{
Ulf Liljenqvist
}

\section{Zusammenfassung}

Die idiopathische Skoliose stellt eine dreidimensionale Wirbelsäulendeformität dar, deren Ätiologie ungeklärt ist. Man unterscheidet strukturell einbogige von den mehrbogigen Skoliosen sowie Thorakalskoliosen von den (Thorako-)Lumbalskoliosen. Aufgrund der Progredienz unbehandelter Skoliosen werden Patienten mit Krümmungen von zwischen 25 und $40^{\circ}$ Cobb-Winkel im Wachstumsalter mit einem Korsett behandelt. Bei progredienten Skoliosen ab 40-50 CobbWinkel ist die operative Korrektur und Instrumentationsspondylodese indiziert. An operativen Verfahren stehen ventrale und dorsale Korrekturtechniken zur Verfügung. Dabei können einbogige Skoliosen entweder vom dorsalen oder ventralen Zugang korrigiert werden, strukturell mehrbogige Skoliosen dagegen werden von dorsal operativ versorgt.

\section{Aetiology, Classification and Therapy of Idiopathic Scoliosis}

Idiopathic scoliosis is a three-dimensional spinal deformity of unknown aetiology. The different curve types are classified into thoracic and thoracolumbar/lumbar scoliosis and into single and multiple curves. Juvenile and adolescent patients with progressive curves with a Cobb angle between 25 and $40^{\circ}$ are treated with a brace. In progressive curves with a Cobb angle exceeding $40-50^{\circ}$, surgical correction and fusion are indicated. Surgical procedures include anterior and posterior correction techniques. Single curves can be treated from an anterior or posterior approach, whereas scolioses with multiple structural curves are treated from the back.

\section{Ätiologie der idiopathischen Skoliose}

Zahlreiche Studien haben sich mit der Ätiologie der idiopathischen Skoliose beschäftigt. Als mögliche Faktoren in der Entstehung der idiopathischen Skoliose hat man genetische, metabolische, endokrine, aber auch zentralnervöse Faktoren diskutiert [10]. Die zunächst vielversprechenden Ergebnisse aus tierexperimentellen Studien zur Rolle des Melatonins in der Pathogenese von Skoliosen haben sich in 1. klinischen Untersuchungen noch nicht bestätigen können [3].
OP-JOURNAL 2009; 25: 132-135

(c) Georg Thieme Verlag KG Stuttgart · New York DOI 10.1055/s-0029-1186023
Man unterscheidet je nach Lokalisation der Primärkrümmung Thorakalskoliosen (Scheitelwirbel Th11 oder höher) von Thorakolumbalskoliosen (Scheitelwirbel Th12 oder L1) und Lumbalskoliosen (Scheitelwirbel L2 oder L3). Während Thorakalskoliosen häufig mit einer $\mathrm{Ab}$ flachung des sagittalen Profils im Sinne eines thorakalen Flachrückens einhergehen, findet sich bei den Thorakolumbalskoliosen eher eine Kyphosierung des thorakolumbalen Übergangs. Skoliosen werden nach der Lenke-Klassifikation klassifiziert. Es werden grundsätzlich 6 Krümmungstypen unterschieden, wobei in strukturelle und nicht strukturelle Krümmungen differenziert wird. Ferner finden das sagittale Profil und speziell auch die Form der lumbalen Krümmung (sog. „lumbar modifier“) Berücksichtigung [6].

Die heutigen Behandlungsrichtlinien ergeben sich insbesondere aus den Erfahrungen älterer Studien zum natürlichen Verlauf unbehandelter idiopathischer Skoliosen. Nachemson u. Mitarb. [11] errechneten die Progredienzwahrscheinlichkeit idiopathischer Skoliosen in Abhängigkeit vom Krümmungsausmaß und fanden bei den 10 - bis 12-jährigen Patienten eine Progredienzwahrscheinlichkeit von $25 \%$ bei Krümmungen unter $20^{\circ}$ Cobb-Winkel, bei Krümmungen zwischen 20 und $29^{\circ}$ Cobb-Winkel lag die Progredienzwahrscheinlichkeit bei $60 \%$ und für Krümmungen von 30 bis $60^{\circ}$ Cobb-Winkel bei $90 \%$. Skoliosen mit einem Cobb-Winkel von mehr als $60^{\circ}$ waren in $100 \%$ progredient. Bei Patienten mit abgeschlossenem Skelettwachstum waren $10 \%$ der Krümmungen zwischen 20 und $29^{\circ}$, 30\% der Krümmungen zwischen 30 und $60^{\circ}$ sowie $70 \%$ der Krümmungen von mehr als $60^{\circ}$ CobbWinkel progredient. Weinstein und Ponseti [13] definierten Risikofaktoren für eine Krümmungsprogredienz auch nach Wachstumsabschluss. Für Thorakalskoliosen waren dies ein Cobb-Winkel von mehr als $50^{\circ}$ und eine Fehlrotation des 


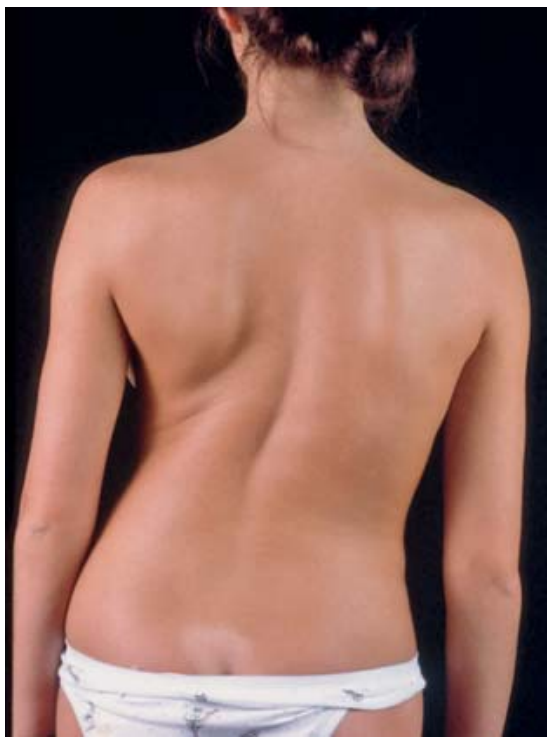

Abb. 1 17-jährige Patientin mit einer rechtskonvexen Thorakalskoliose. Ausgeprägte Rumpfasymmetrie mit Rumpfüberhang und Schulterschiefstand sowie asymmetrischen Taillendreiecken.

Scheitelwirbels von mehr als $30^{\circ}$. Die Risikofaktoren für lumbale Skoliosen waren ein Cobb-Winkel und eine Fehlrotation von jeweils mehr als $30^{\circ}$.

Die Krümmungsprogredienz allein ist jedoch nicht das einzige Problem unbehandelter Skoliosen.

So gehen höhergradige Thorakalskoliosen mit einer vermehrten Rechtsherzbelastung und den möglichen Folgen eines Cor pulmonale einher.

Thorakolumbale und lumbale Krümmungen können zu vermehrten Kreuzschmerzen führen und auch die psychosozialen Folgen der kosmetischen Beeinträchtigung sind nicht unerheblich.

\section{Konservative Therapie}

Die konservative Therapie der idiopathischen Skoliose umfasst die krankengymnastische Übungs- und die Korsettbehandlung.

Die Elektrostimulation hat sich in prospektiven, randomisierten Studien als nicht effektiv erwiesen und sollte nicht mehr durchgeführt werden [12]. Es gibt zahlreiche krankengymnastische Therapiekonzepte zur Behandlung idiopathischer Skoliosen. Dabei hat sich insbesondere die dreidimensionale Skoliosebehandlung nach Katharina Schroth als eine bewährte Technik zur Aufrichtung

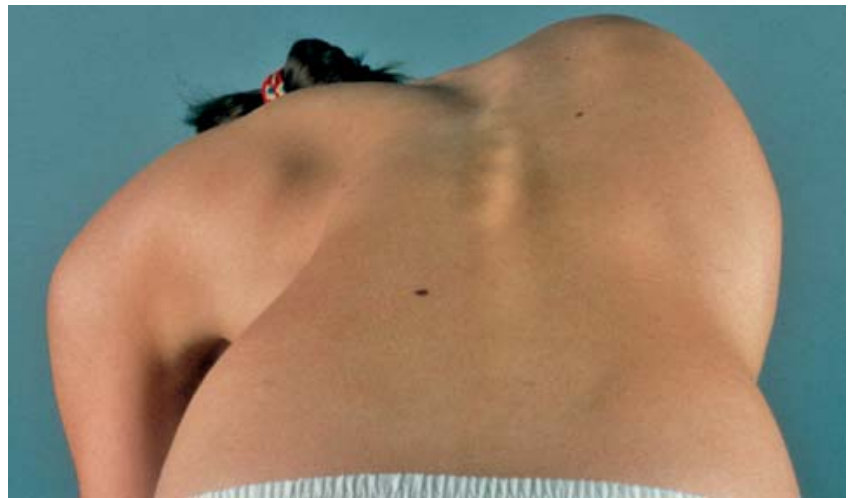

Abb. 2 In der Vornüberneige ist bei höhergradigen Thorakalskoliosen der typische Rippenbuckel zu erkennen.

der skoliotischen Fehlhaltung etabliert. Allerdings fehlen bis heute prospektive, randomisierte Studien, die eine Wirksamkeit der krankengymnastischen Übungsbehandlung gegenüber dem natürlichen Verlauf belegen können.

Bez. der Effektivität der Korsettbehandlung gibt es kontroverse Ansichten. Nachemson und Peterson [12] konnten in einer prospektiven Multicenterstudie zeigen, dass die mit dem Korsett behandelten Skoliosen eine signifikant geringere Progredienz aufwiesen als die unbehandelten Patienten. Dagegen konnten Goldberg u. Mitarb. [4] in ihrer Studie keine signifikanten Unterschiede hinsichtlich der Progredienz von mit dem Korsett behandelten und unbehandelten Skoliosen finden. Bisher liegen prospektive, randomisierte Studien zur Effektivität der Korsettbehandlung nicht vor.

Dennoch gilt die Korsettbehandlung bei nicht ausgewachsenen Patienten mit Skoliosen von zwischen 25 und $40^{\circ}$ Cobb-Winkel nach wie vor als die Standardtherapie. Dabei ist das Korsett 23 Stunden täglich zu tragen und die Rumpfmuskulatur regelmäßig krankengymnastisch zu beüben.

Neben der klinischen Verlaufskontrolle ermöglicht die Videorasterstereografie eine dreidimensionale Analyse der Wirbelsäulendeformität (Abb. 3).

Durch ihren routinemäßigen Einsatz ist es uns gelungen, die Anzahl der Röntgenuntersuchungen drastisch zu senken. In unserer prospektiven Korsettstudie an über 70 Patienten konnten wir zeigen, dass nach zunächst etwa 50\%iger Krümmungskorrektur durch das Korsett die Krümmungen nach Korsettabschulung im Durchschnitt wieder die Krümmungsausmaße vor der Behandlung erreichen. In 33\% waren die Skoliosen trotz

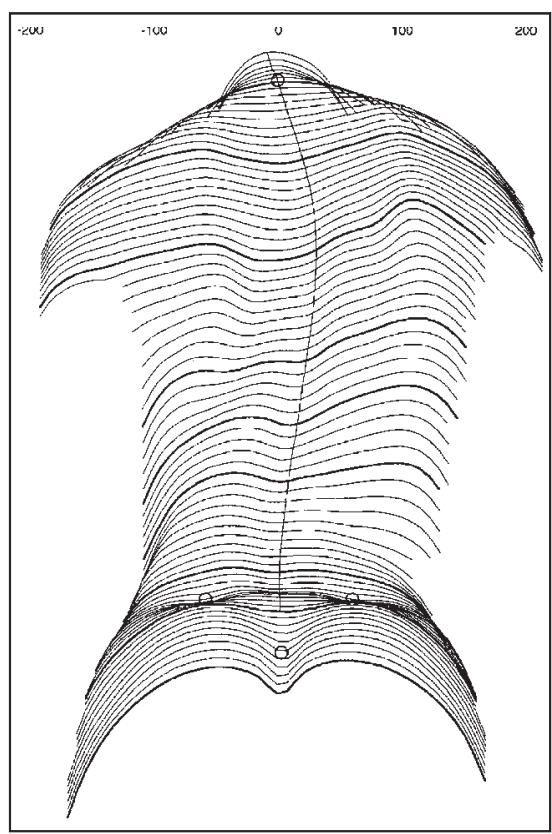

Abb. 3 In der Videorasterstereografie kann die Wirbelsäulendeformität dreidimensional analysiert werden.

Korsettbehandlung progredient [1]. Als Risikofaktoren für eine Krümmungsprogredienz gelten dabei die Skolioseentstehung vor dem 10. Lebensjahr (juvenile Skoliosen), eine hohe Ausgangskrümmung, eine späte Menarche, eine schlechte Krümmungskorrektur im Korsett und eine thorakale Krümmung [8].

\section{Operative Therapie}

\section{Indikationen, Zielsetzung}

Indikationen zur operativen Therapie idiopathischer Skoliosen sind progrediente Krümmungen ab 40-50 CobbWinkel. Dabei spielen jedoch Faktoren wie Patientenalter, Skoliosetyp und auch der Grad der kosmetischen Beeinträchtigung eine wichtige Rolle in der Indikationsstellung zur operativen Therapie. 
Ziele der operativen Behandlung sind eine optimale dreidimensionale Krümmungskorrektur mit lotgerechter Ausrichtung der Wirbelsäule in sagittaler und frontaler Ebene, ein gutes kosmetisches Ergebnis mit maximaler Reduktion des Rippenbuckels und Sicherstellung eines Schultergeradstands, eine möglichst kurze Fusionsstrecke und eine primär stabile Instrumentation, die eine korsettfreie Nachbehandlung ermöglicht (Abb. 4 und 5).

Im Rahmen der operativen Formkorrektur wird die Wirbelsäule zunächst mit unterschiedlichen Verankerungstechniken korrigiert und im Sinne einer Instrumentationsspondylodese stabilisiert. Dies kann von ventral oder auch dorsal erfolgen. Bei schwergradigen Skoliosen kommen auch kombiniert ventrodorsale Instrumentationsspondylodesen zur Anwendung.

\section{Dorsale Instrumentationsspondylodesen}

Durch die Einführung des Cotrel-Dubousset-Instrumentariums in den 80erJahren und die Etablierung der segmentalen Pedikelschraubenkorrektur in den 90er-Jahren hat sich die dorsale Skoliosechirurgie maßgeblich weiterentwickelt [7]. Dabei erfolgt die Krümmungskorrektur über Translation, Derotation sowie Distraktion und Kompression mit einem Doppelstabsystem, das mit überwiegend Pedikelschrauben an der Wirbelsäule fixiert wird (Abb.6). Nach erfolgter Korrektur werden die dorsalen knöchernen Strukturen im Instrumentationsbereich mit dem Meißel angefrischt und Knochen angelegt, um eine knöcherne Durchbauung (Spondylodese) zu erzielen.

Erst die knöcherne Versteifung sichert langfristig das Korrekturergebnis.

Die Hauptindikation zur dorsalen Skoliosekorrektur sind doppelbogige Skoliosen mit mehreren strukturellen Krümmungen.

Aber auch einbogige Skoliosen werden von vielen Autoren von dorsal operiert. Einbogige Thorakolumbalskoliosen mit Krümmungswerten bis $\mathrm{zu} 60^{\circ}$ können bei einer mit ventralen Techniken vergleichbaren Fusionsausdehnung von dorsal instrumentiert werden [5]. Bei Thorakalskoliosen wird häufig die dorsale Korrektur und Fusion mit einer Rippenbuckelresektion kombiniert, die kosmetisch sehr gute Ergebnisse liefert. Inwieweit die Rotation bei den dorsalen Operationstechniken korrigiert werden

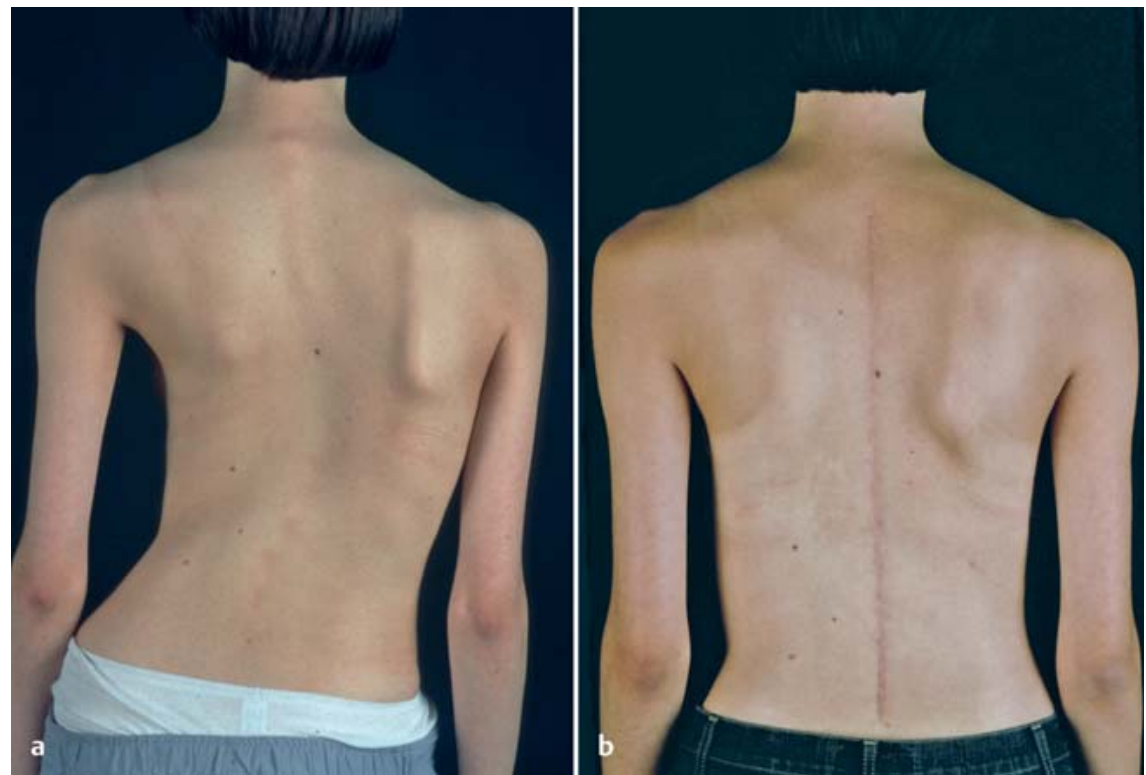

Abb.4a und b Prä- und postoperative klinische Bilder einer 14-jährigen Patientin, deren Thorakalskoliose vom hinteren Zugang korrigiert und stabilisiert wurde. Zu beachten ist die Wiederherstellung der Rumpfsymmetrie.
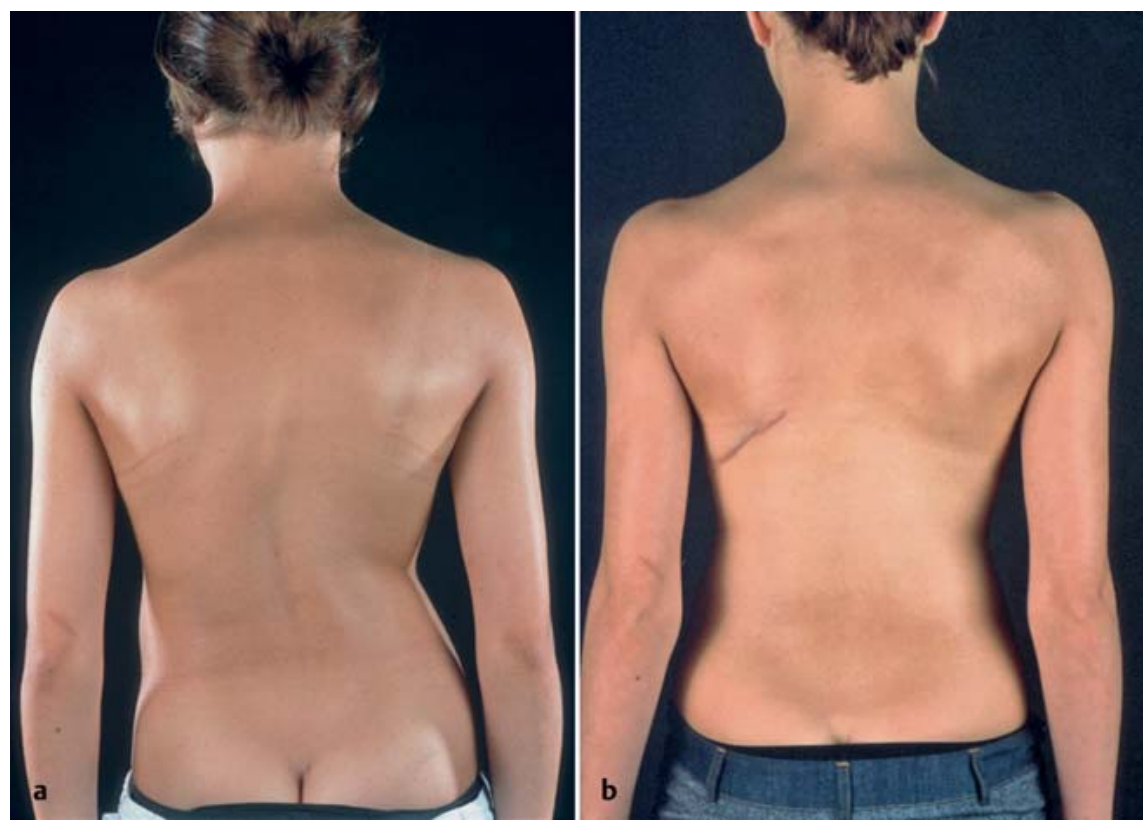

Abb.5a und b Prä- und postoperative klinische Bilder einer 15-jährigen Patientin, deren Thorakolumbalskoliose vom seitlichen Zugang korrigiert und stabilisiert wurde. Auch hier hat die operative Korrektur zu einer Wiederherstellung der Rumpfsymmetrie geführt.

kann, ist umstritten. Die Korrektur des Cobb-Winkels (also der frontalen Ebene) beträgt je nach Flexibilität der Krümmung zwischen 50 und $80 \%$. Auch das sagittale Profil kann vom dorsalen $\mathrm{Zu}$ gang effektiv beeinflusst werden [5].

\section{Ventrale Instrumentationsspondylodesen}

Dwyer und Zielke haben die 1. ventralen Wirbelsäulensysteme zur Skoliosekor- rektur entwickelt. Die Vorteile insbesondere der ventralen Derotationsspondylodese nach Zielke sind die kurze Fusionsstrecke und die gute Derotation und Korrektur in der frontalen Ebene. Die Nachteile der fehlenden Primärstabilität sowie der mangelnden Kontrolle des sagittalen Profils konnten im Rahmen von Weiterentwicklungen eliminiert werden [9]. 
Die Indikation zur ventralen Skoliosekorrektur sind insbesondere die Lumbal-und die Thorakolumbalskoliosen (Abb. 7).

Neben der kurzen Fusionsstrecke und der guten Korrektur in der frontalen und sagittalen Ebene ist hier insbesondere die gute Derotation von im Durchschnitt $50 \%$ hervorzuheben. In der Frontalebene werden für diese Form der Skoliose Korrekturwerte von zwischen 60 und 80\% angegeben. In den letzen Jahren haben sich ventrale Korrekturtechniken jedoch auch in der operativen Behandlung der Thorakalskoliose etabliert. Die entscheidenden Vorteile gegenüber dorsalen Instrumentationen sind die kürzere Fusionsstrecke und die bessere Wirbelderotation. Durch moderne Implantatsysteme konnte auch der zuletzt noch für thorakale Krümmungen bestehende Nachteil der fehlenden Primärstabilität eliminiert werden, sodass auch in diesen Fällen die Patienten postoperativ korsettfrei nachbehandelt werden können [9].

\section{Ventrodorsale Instrumentations- spondylodesen}

Bei schwerstgradigen rigiden Skoliosen (Cobb-Winkel mehr als $90^{\circ}$ ) ist eine kombinierte Vorgehensweise erforderlich, um ein gutes Korrekturergebnis zu bekommen. Dabei wird die Wirbelsäulendeformität zunächst von ventral gelöst und teilkorrigiert und anschließend in gleicher Sitzung von dorsal korrigiert und instrumentiert. In besonders schwergradigen und rigiden Fällen wird z.T. auch zunächst dorsal gelöst (einschließlich multiplen Rippenosteotomien) und dann von ventral und schließlich von dorsal korrigiert und instrumentiert (dorsoventrodorsale Vorgehensweise). Durch dieses aggressive chirurgische Vorgehen kann auf die für den Patienten stark belastende Haloextensionsbehandlung in aller Regel verzichtet werden. Die Korrekturwerte für den Cobb-Winkel liegen im Durchschnitt bei etwa $60 \%$ und auch die kosmetischen Ergebnisse sind als sehr gut zu werten [2].

\section{Rippenbuckelresektion}

Bei kosmetisch störendem Rippenbuckel kann durch eine gezielte Resektion der prominenten Rippenanteile der Rippenbuckel deutlich abgesenkt und somit das kosmetische Ergebnis der Operation weiter verbessert werden. Dabei kann die Rippenbuckelresektion sowohl im Rahmen eines ventralen als auch eines dorsalen Eingriffs jeweils über den glei-

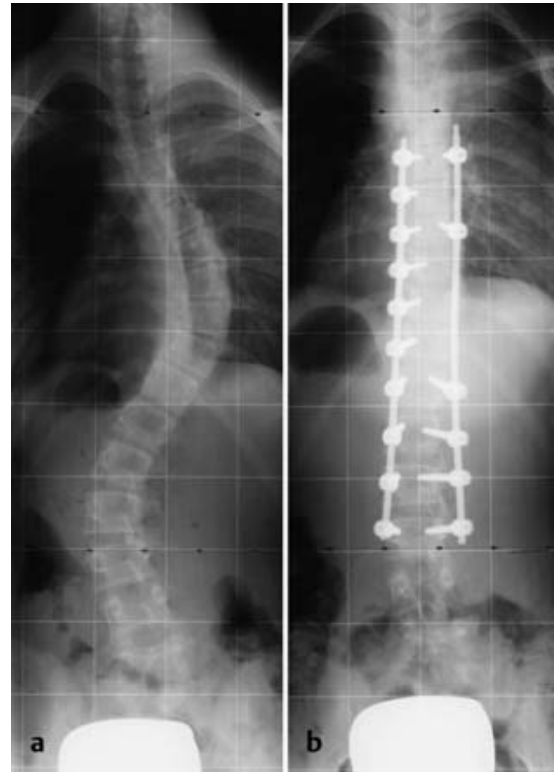

Abb. $6 \mathbf{a}$ und b Prä- und postoperative Röntgenbilder einer 14-jährigen Patientin mit einer doppelbogigen Skoliose. Es erfolgte die dorsale Korrektur und Stabilisierung mittels segmentaler Pedikelschraubeninstrumentation.

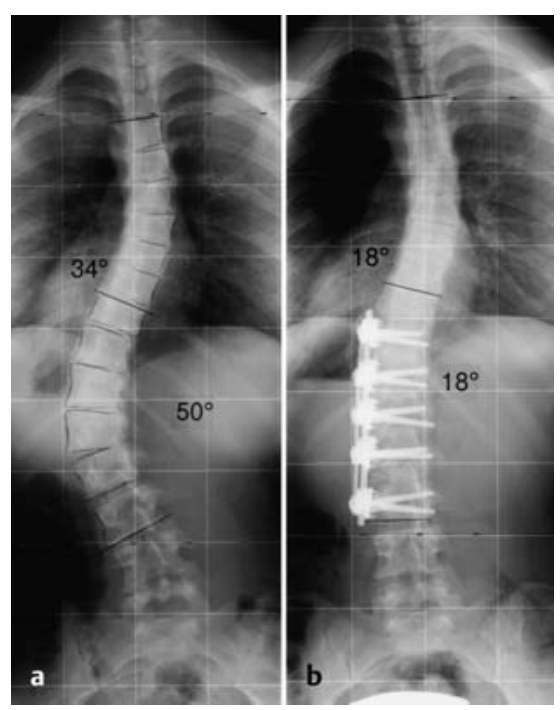

Abb. 7 a und b Prä- und postoperative Röntgenbilder einer 15-jährigen Patientin mit einer Thorakolumbalskoliose. Es erfolgte die ventrale Korrektur und Stabilisierung mittels Doppelstabinstrumentation.

chen Hautschnitt durchgeführt werden. Durch die Gewinnung zusätzlicher autologer Knochenspäne von den resezierten Rippenanteilen kann in diesen Fällen auf eine Beckenkammspanentnahme verzichtet werden.

\section{Schlussfolgerung}

Die idiopathische Skoliose stellt eine dreidimensionale Wirbelsäulendeformität ungeklärter Ätiologie dar, die ins- besondere im Jugendalter auftritt. Unbehandelt kann sie bei Progredienz zu Funktionseinschränkungen von Herz und Lunge, zu Rückenschmerzen und kosmetischer Beeinträchtigung führen. Skoliosen mit einem Winkel von zwischen 25 und $40^{\circ}$ werden konservativ in einem redressierenden Korsett bis zum Wachstumsabschluss behandelt. Progrediente Skoliosen ab 40-50 sollten operativ korrigiert und stabilisiert werden. Dabei kommen ventrale wie auch dorsale Operationstechniken zur Anwendung.

\section{Literatur}

1 Bullmann V, Halm H, Lerner $T$ et al. Die prospektive Korsettstudie Münster. Z Orthop 2004; 14: 403-409

2 Bullmann V, Halm H, Schulte T et al. Combined anterior and posterior instrumentation in severe and rigid idiopathic scoliosis. Eur Spine J 2006; 15: 440-448

${ }^{3}$ Fagan A, Kennaway D, Sutherland A. Total 24hour melatonin secretion in adolescent idiopathic scoliosis. Spine 1998; 23: 41-46

4 Goldberg C, Dowling F, Hall F et al. A statistical comparison between natural history of idiopathic scoliosis and brace treatment in skeletally immature adolescent girls. Spine 1993; 18: 902-908

${ }^{5}$ Halm H, Niemeyer T, Link $T$ et al. Segmental pedicle screw instrumentation in idiopathic thoracolumbar and lumbar scoliosis. Eur Spine J 2000; 9: 191-197

${ }^{6}$ Liljenqvist $U$, Lerner T, Bullmann V. Selektive Fusion idiopathischer Skoliosen unter Berücksichtigung der Lenke Klassifikation. Orthopäde 2009; 38: 194-207

${ }^{7}$ Liljenqvist $U$, Halm $H$, Link T. Pedicle screw instrumentation of the thoracic spine in idiopathic scoliosis. Spine 1997; 22: 2239-2245

8 Liljenqvist U, Niemeyer T, Grävinghoff $T$ et al. Conservative treatment of idiopathic scoliosis with the Cheneau brace. J Bone Joint Surg [Br] 1998; 80: 241

9 Liljenqvist $U$, Bullmann $V$, Schulte T et al. Anterior dual rod instrumentation in idiopathic thoracic scoliosis. Eur Spine J 2006; 15: 1118-1127

10 Machida $M$. Cause of idiopathic scoliosis. Spine 1999; 15: 2576-2583

11 Nachemson A, Lonstein J, Weinstein S. Report of the Scoliosis Research Society Prevalence and Natural History Committee; 1982

12 Nachemson A, Peterson L. Effectiveness of treatment with a brace in girls who have adolescent idiopathic scoliosis. J Bone Joint Surg [Am] 1995; 77: 815-822

13 Weinstein S, Ponseti I. Curve progression in idiopathic scoliosis: long-term follow-up and prognosis in untreated patients. J Bone Joint Surg [Am] 1983; 65: 447-453

\section{Prof. Dr. med. Ulf Liljenqvist}

Chefarzt der Orthopädischen Klinik II, Wirbelsäulenchirurgie

St. Franziskus Hospital Hohenzollernring 72 48145 Münster

ulf.liljenqvist@sfh-muenster.de 